Supporting Information for

\title{
Synthesis of the NK1 Receptor Antagonist GW597599. Part 1: Development of a Scalable Route to a Key Chirally Pure
}

\section{Arylpiperazine}

Giuseppe Guercio*, Sergio Bacchi*, Michael Goodyear, Antonella Carangio, Francesco Tinazzi, Stefano Curti.

Department of Chemical Development, GlaxoSmithKline, Via Fleming 4, 37135, Verona, Italy

\section{Process Safety Evaluation of bromo-(4-fluoro-2-methylphenyl)magnesium 19}

The proposed procedure for the bromo-(4-fluoro-2-methylphenyl)magnesium 19 formation was investigated from a process safety perspective to ensure that sound basis of safety existed prior to pilot plant scale up.

A desk screening suggested that the main issues were the thermal stability of the Grignard itself and the probable induction period of the Grignard formation reaction, which could result in an accumulation of the reagents and eventually in a sudden, large exothermic event followed by a rapid temperature increase inside the vessel and possibly in violent solvent boiling or secondary exothermic decomposition. A preliminary assessment of the thermal stability of the Grignard reagent by Differential Scanning Calorimetry (DSC) showed that the solution of the Grignard reagent was stable at the process temperature. After that, a deeper investigation was carried out, in particular by means of a coupled Thermogravimetry-Single Differential Thermal Analisys (TGA-SDTA) technique. This allowed revealing that the desired Grignard reagent may decompose even at room temperature when its solution is concentrated. Thus the bromo-(4-fluoro-2-methylphenyl)magnesium 19 formation reaction was studied in a reaction calorimeter. These experiments confirmed that the initiation was a key step to ensure a safe scale up for this process. The heat release associated with the Grignard formation reaction would have been capable of rising the temperature of the reaction mixture of more than $200^{\circ} \mathrm{C}$ if the reaction would have been carried out under adiabatic conditions. The addition of the whole 2-bromo-5-fluorotoluene before the confirmation of the reaction initiation could have resulted in an accumulation of the 2-bromo-5-fluorotoluene in

\footnotetext{
* To whom correspondence should be addressed. E-mail: giuseppe.2.guercio@gsk.com, sergio.k.bacchi@gsk.com
} 
solution and in a sudden, rapid reaction that would approximate adiabatic conditions consequently resulting in solvent boil over and possible thermal decomposition of the Grignard reagent. A basis of safety for this process was imposing limits to the process parameters before initiation confirmation via HPLC analysis. In particular, the reaction temperature was kept at or below $40^{\circ} \mathrm{C}$ and no more than $8 \%$ of the overall 2-bromo-5fluorotoluene was added prior to reaction initiation.

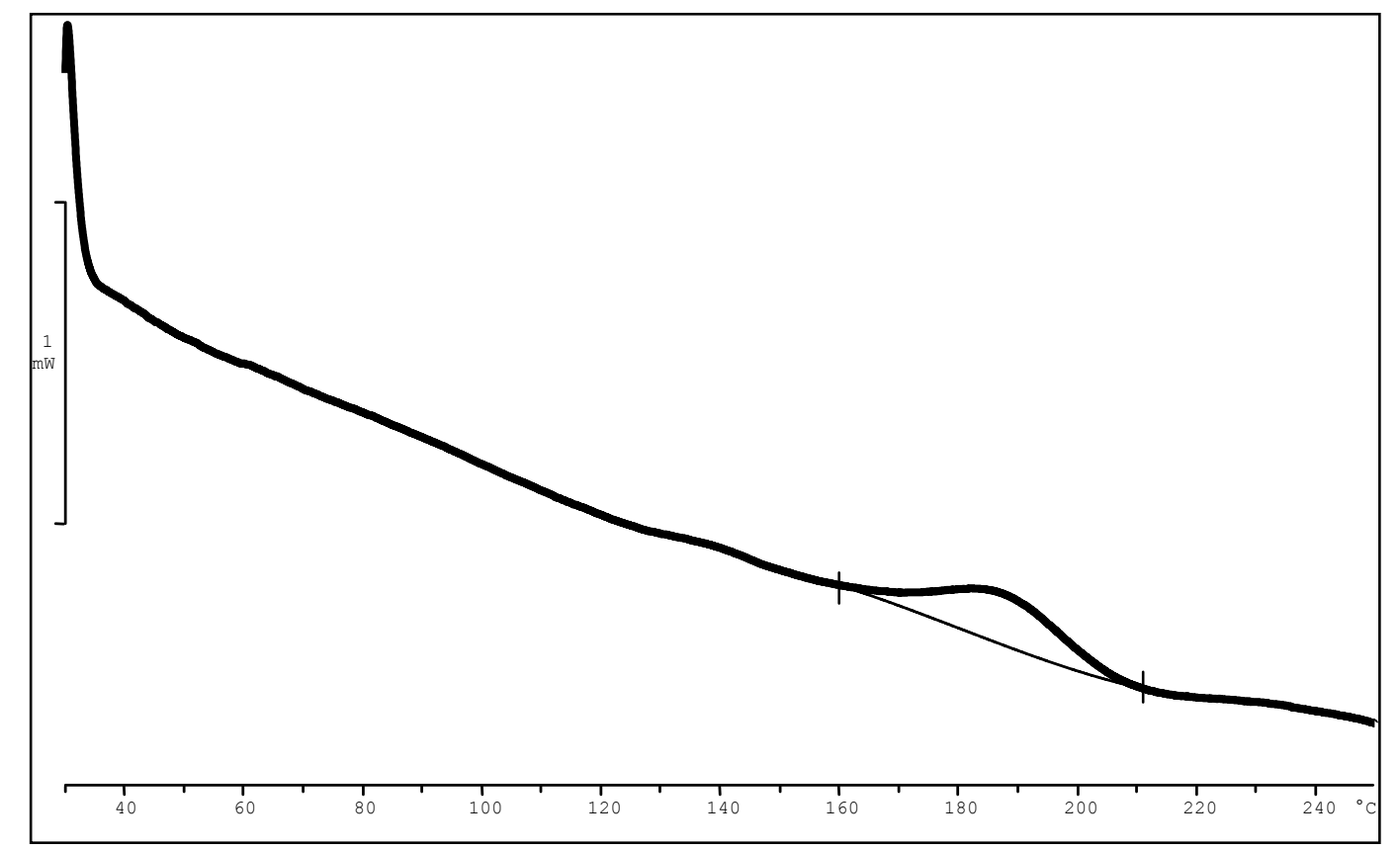

Figure 1 Thermal stability evaluation of bromo-(4-fluoro-2-methylphenyl)magnesium solution. The plot is a DSC trace showing an exothermic peak due to thermal decomposition starting from $c a .160^{\circ} \mathrm{C}$. 


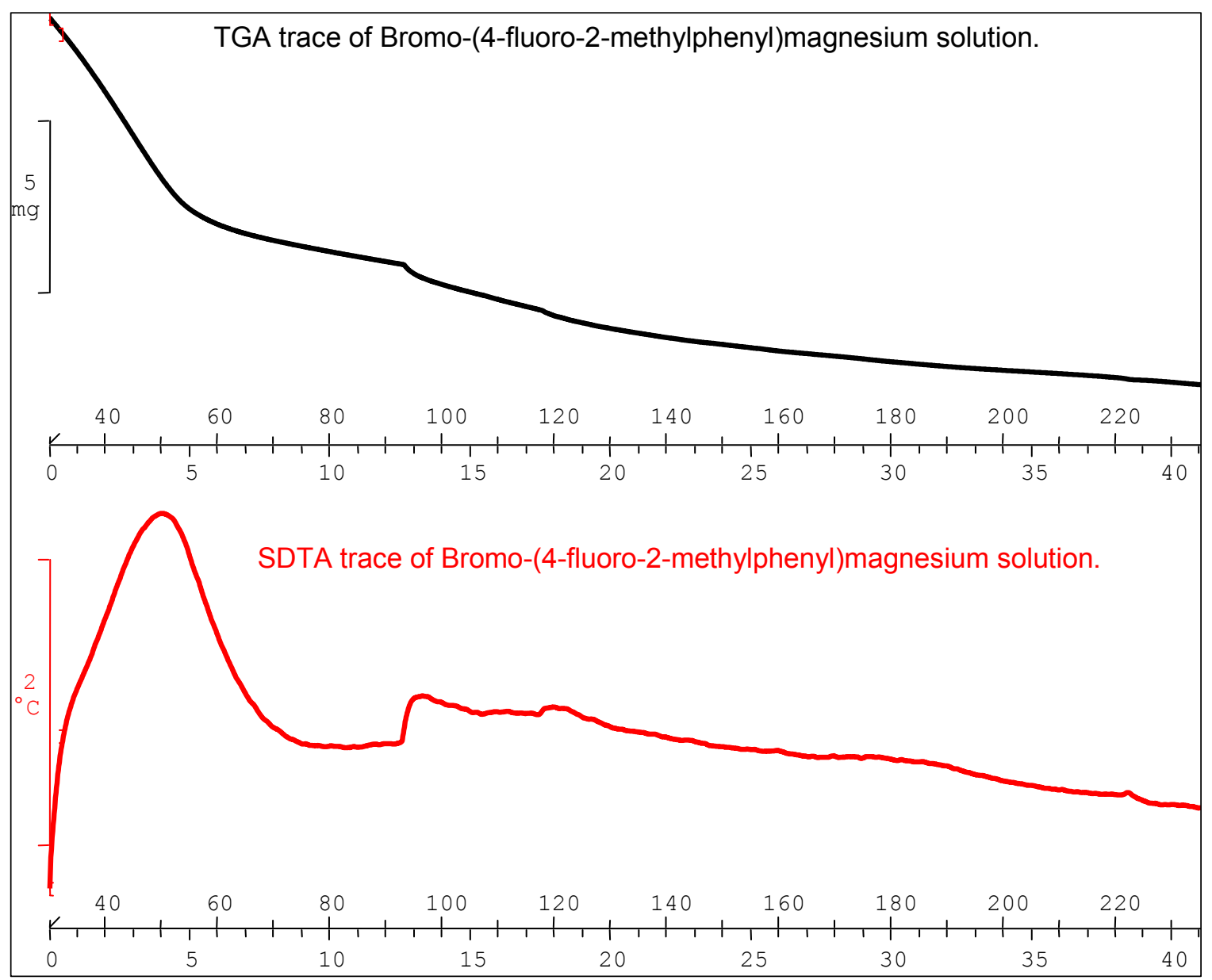

Figure 2 Thermal stability evaluation of bromo-(4-fluoro-2-methylphenyl)magnesium solution. The plot refers to a coupled TGA - SDTA experiment. The sample has been heated in an open pan, thus resulting in rapid solvent evaporation and so in weight loss (see TGA trace). The SDTA trace shows an exothermic peak due to thermal decomposition starting from ca. $35^{\circ} \mathrm{C}$. This confirmed that the Grignard solution becomes more unstable upon concentration. 


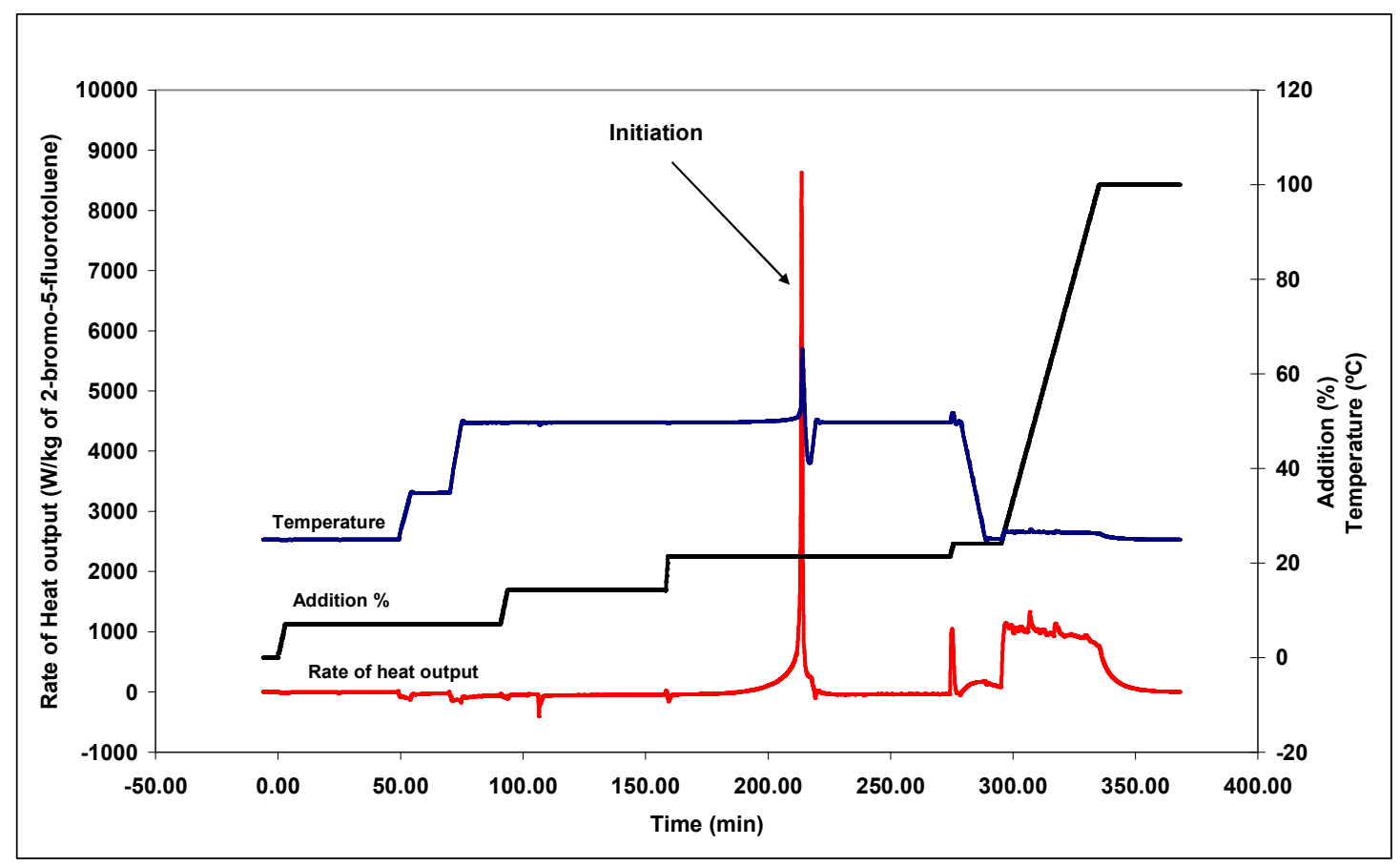

Figure 3 Reaction calorimetry evaluation of the formation of bromo-(4-fluoro-2methylphenyl)magnesium. The plot shows that heating to $50^{\circ} \mathrm{C}$ and the addition of 3 portions of 2-bromo-5-fluoro toluene, corresponding to $20 \%$ of the total charge, were necessary to initiate the Grignard formation in $c a$. 3hrs. The addition of the remaining $80 \%$ of 2-bromo-5fluoro toluene resulted in a controllable heat generation. A basis of safety for this process was identified by imposing a maximum temperature of $40^{\circ} \mathrm{C}$ and a maximum amount of 2-bromo5-fluoro toluene corresponding to $8 \%$ of the overall charge before the reaction initiation thus avoiding violent solvent boiling and thermal decomposition of the Grignard reagent. Grignard formation initiation was also confirmed by HPLC analysis. 
Chiral HPLC of (3S)-3-(4-Fluoro-2-methylphenyl)-2-piperazinone 14.

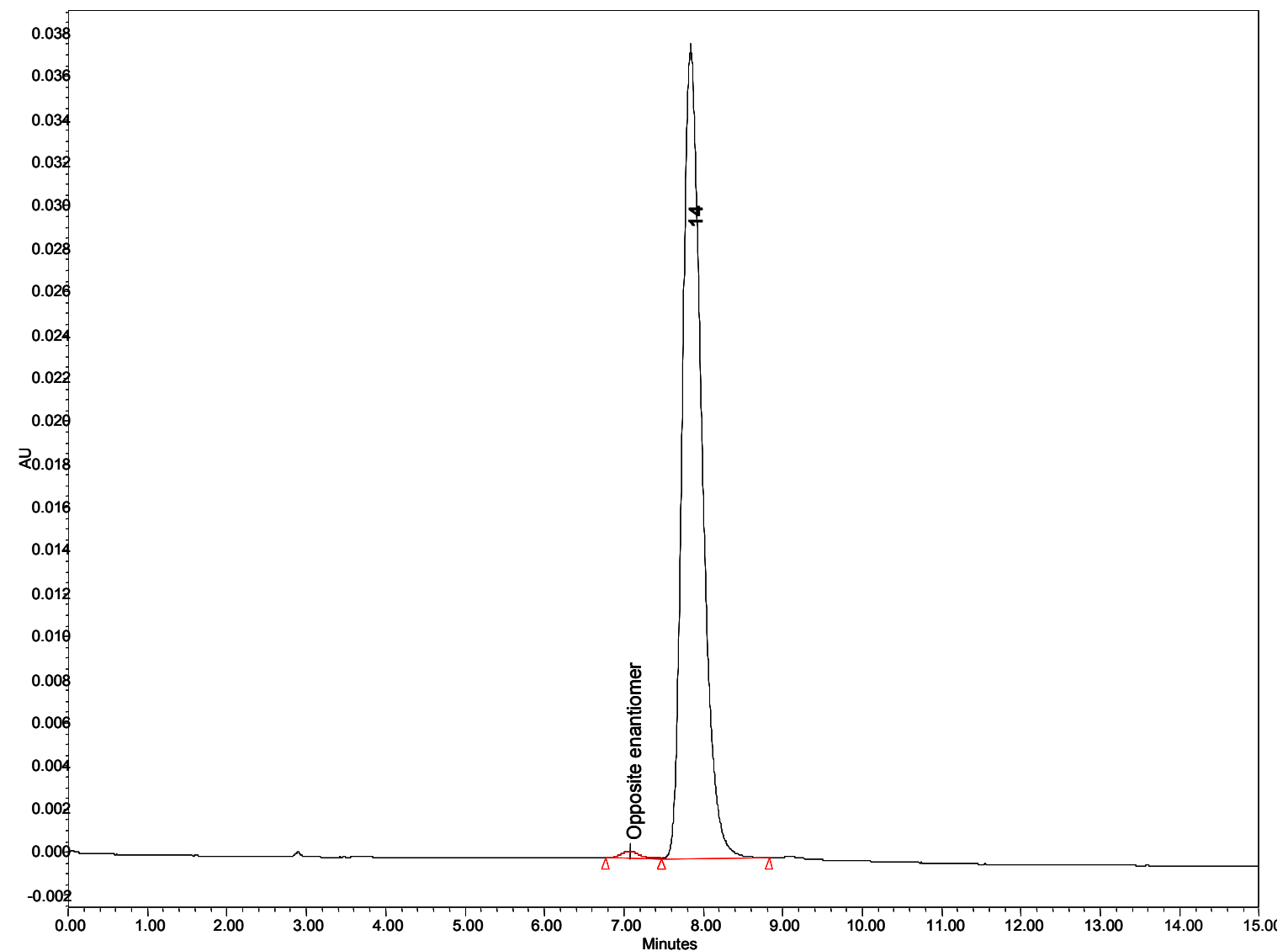

